\title{
Polaron Dynamics with Impurities in Conjugated Polymers
}

\author{
M. P. Lima and G. M. e Silva \\ Institute of Physics, University of Brasília, 70.917-970, Brasília, Distrito Federal, Brazil
}

Received on 3 December, 2004

\begin{abstract}
We study the charge transport by polarons in conjugated polymers in the presence of impurities. The effects on the polaron motion due to the symmetry of the chain is considered. The polaron dynamics in doped conjugated polymers is numerically studied using the Su-Schrieffer-Heeger (SSH) model combined with the Pariser-ParrPople (PPP) model modified to include an external electric field and the Brazovskii-Kirova (BK) symmetry breaking interaction. The time dependent Hartree-Fock approximation is used. The electric field is used to accelerate the polaron in a polymer chain. Polaron trapped by the impurity and polaron-impurity collisions are considered. When the polaron-structure is around the impurity site a charge oscillation can be observed. Nevertheless, when there are collisions between the polaron and the impurity, the polaron-structure can pass, be trapped or be reflected. These effects are determined by the strength of the radical parameter and by the electric field intensity. The effects on the polaron are analyzed and an effective potential is determined. Therefore, the effective potential determines the polaron behavior for each case, as the polaron pass, or it is trapped or reflected by an impurity.
\end{abstract}

\section{INTRODUCTION}

In the scientific technological revolution of the last few years the study of high-performance materials has been increasing and steady. The development of molecular devices allows the creation of new architectures for electronic components. The research of more powerful and small electronic devices opens the possibility of finding devices with new and important features.

Recent works have showed that conducting polymers have special properties that are interesting of the point of view of this new technology [1-5]. Conjugated polymers are good materials to be employed in the fabrication of molecular electronic devices because they have properties that can be controlled by external parameters. The charge carriers in conjugated polymers are a fundamental part of this new technological search. In conjugated polymers, nonlinear excitations, such as solitons, polarons and bipolarons are essential to describe the charge transport.

Polarons are charge carriers generated upon doping or photo-excitation in conjugated polymers with charge $Q= \pm e$ and spin $\pm \frac{1}{2}$. In this paper we concentrate ourselves in discussing effects on polarons due to doping interactions in conjugated polymers. The purpose of the present work is to establish which parameters rule the polaron dynamics in doped conjugated polymers. The study of polaron properties is made through numerical solutions of equations of motion for the lattice and electronic degrees of freedom [6-10]. The initial polaron configuration has been generated by self consistent equations. The electric field is used in order to accelerate the polaron structure.

\section{MODEL}

The difference between symmetric and asymmetric configurations is given by a Brazovskii-Kirova symmetry breaking interaction [7] (considering a symmetry breaking parameter $\left.\delta_{0}\right)$. The structural differences are shown in Fig. 1. For both

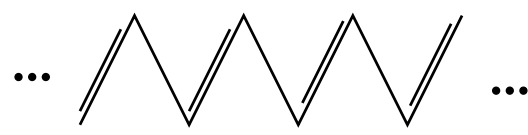

(a) symmetric chain

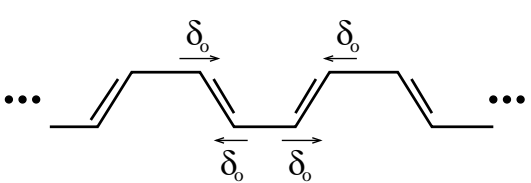

(b) asymmetric chain

FIG. 1: (a) Symmetric chain. (b) asymmetric chain. The symmetry breaking is introduced with a BK-symmetry breaking term $\delta_{0}$.

cases we perform collisions between polarons and impurities. In this work we use single-site impurities.

The polaron structure can be localized close to an impurity. When the electric field is applied, the polaron can move in the polymer lattice or be trapped on the impurity. The condition for the polaron to untrap and to run in the polymer depends on the electric field intensity, the time spans which it remains turned on and the strength of the impurity's parameter. When the polaron is trapped in an impurity, the site of this impurity does not coincide with the polaron center position. The displacement between the polaron position and the impurity site depends on the strength of the impurity.[11]

The Hamiltonian of the SSH model has been extended in order to include an external electric field, combined with PPP model, with BK-symmetry interactions and a site-type impurity term: 


$$
\begin{aligned}
H(t) & =-\sum_{i, s}\left(t_{i, i+1} C_{i+1, s}^{\dagger} C_{i, s}+H . c\right) \\
& +U \sum_{i}\left(n_{i \uparrow}-\frac{1}{2}\right)\left(n_{i \downarrow}-\frac{1}{2}\right)+V \sum_{i}\left(n_{i}-1\right)\left(n_{i+1}-1\right) \\
& +\sum_{i} \frac{K}{2} y_{i}^{2}+\sum_{i} \frac{M}{2} \dot{u}_{i}^{2}+\sum_{p, s} V_{p} C_{p, s}^{\dagger} C_{p, s},
\end{aligned}
$$

where $C_{i, s}^{\dagger}\left(C_{i, s}\right)$ is the creation (annihilation) operator of a $\pi$ electron with spin $s$ at the $i$ th lattice site, $n_{i, s} \equiv C_{i, s}^{\dagger} C_{i, s}$ is the number operator, and $n_{i}=\sum_{s} n_{i, s}, y_{n} \equiv u_{n+1}-u_{n}$, where $u_{n}$ is the displacement of the $n$th $\mathrm{CH}$-group from equilibrium position in the undimerized phase. $t_{n, n+1}=\exp (-i \gamma A)[(1+$ $\left.\left.(-1)^{n} \delta_{0}\right) t_{0}-\alpha y_{n}\right], t_{0}$ is the transfer integral between the nearest neighbour sites in the undimerized chains, $\alpha$ is the electron-phonon coupling, $\delta_{0}$ is the BK symmetry-breaking parameter. In the case of lattice without symmetry breaking interaction $\delta_{0}=0 . \quad \mathrm{M}$ is the mass of a $\mathrm{CH}$ group, $K$ is the spring constant of a $\sigma$-bond, $U$ and $V$ are the on-site and nearest-neighbour Coulomb repulsion strengths, respectively. $V_{p}$ is the strength of an impurity which is located in the $p$ th site, $\gamma \equiv e a /(\hbar c), e$ being the absolute value of the electronic charge, $a$ the lattice constant, and $c$ the light velocity. The relation between the time-dependent vector potential $A$ and the uniform electric field $E$ is given by $E=-\frac{1}{c} \dot{A}$. We use as parameters the commonly accepted values for conjugated polymers: $t_{0}=2.5 \mathrm{eV}, K=21 \mathrm{eV} \AA^{-2}, \alpha=4.1 \mathrm{eV} \AA^{-1}$, $U=t_{0} / 2, V=U / 2, a=1.22 \AA$, and a bare optical phonon energy $\hbar \omega_{Q}=\sqrt{4 H / M}=0.16 \mathrm{eV}$.

We calculate an initial self-consistent state and integrate the Schrödinger equation to perform the time evolution of the system. Firstly, we prepare a stationary state, which is fully self-consistent with the degrees of freedom of electrons and phonons. The Hamiltonian is dependent of degrees of freedom of the electrons and the phonons. There are two equations which have to be consistent with themselves. With the Time Unrestricted Hartree-Fock Approximation we find the following equations for one-particle wave functions:

$$
\begin{aligned}
i \hbar \dot{\psi}_{k, s}(i, t) & =-\left[t_{i, i+1}^{*}+V \tau_{s}(i, t)\right] \psi_{k, s}(i+1, t) \\
& -\left[t_{i-1, i}+V \tau_{s}^{*}(i-1, t)\right] \psi_{k, s}(i-1, t) \\
& +\left\{U\left[\rho_{-s}(i, t)-\frac{1}{2}\right]+\sum_{p} V_{p} \delta_{i, p}\right. \\
& \left.+V \sum_{s^{\prime}}\left[\rho_{s^{\prime}}(i+1, t)+\rho_{s^{\prime}}(i-1, t)-1\right]\right\} \psi_{k, s}(i, t)
\end{aligned}
$$

with

$$
\begin{array}{r}
\rho_{s}(i, t)=\sum_{k}^{\prime} \psi_{k, s}^{*}(i, t) \psi_{k, s}(i, t) \\
\tau_{s}(i, t)=\sum_{k}^{\prime} \psi_{k, s}^{*}(i+1, t) \psi_{k, s}(i, t)
\end{array}
$$

The $\mathrm{CH}$ sites dynamics is given by the Euler-Lagrange equations,

$$
\frac{d}{d t}\left(\frac{\partial\langle L\rangle}{\partial \dot{w}_{n}}\right)-\frac{\partial\langle L\rangle}{\partial w_{n}}=0
$$

where

$$
\langle L\rangle=\langle T\rangle-\langle V\rangle
$$

We obtain

$$
M \ddot{u}_{n}=F_{n}(t)
$$

with

$$
\begin{aligned}
F_{n}(t)= & -K\left[2 u_{n}(t)-u_{n+1}(t)-u_{n-1}(t)\right] \\
& +\alpha\left[e^{i \gamma A(t)}\left(B_{n, n+1}-B_{n-1, n}\right)\right. \\
& \left.+e^{-i \gamma A(t)}\left(B_{n+1, n}-B_{n, n-1}\right)\right] .
\end{aligned}
$$

Here $B_{n, n^{\prime}} \equiv \sum_{k, s}{ }^{\prime} \psi_{k, s}^{*}(n, t) \psi_{k, s}\left(n^{\prime}, t\right)$. The prime on the summation means that the sum is taken over the occupied singleparticle states in the initial stationary state. These equations of motion are solved by discretizing the time variable with a step $\Delta t$. The time step, $\Delta t$, is chosen so that the change of $u_{i}(t)$ and $A(t)$ during this interval is always very small in the electronic scale.

The time-dependent Schrödinger equation is integrated by introducing single-particle eigenstates at each moment. The solutions of the time dependent Schrödinger equation can then be put in the form,

$\psi_{k, s}\left(n, t_{j+1}\right)=\sum_{l}\left[\sum_{m} \phi_{l, s}^{*}\left(m, t_{j}\right) \psi_{k, s}\left(m, t_{j}\right)\right] e^{-i \frac{\varepsilon_{l} \Delta t}{\hbar}} \phi_{l, s}\left(n, t_{j}\right)$,

where $\left\{\phi_{l}\right\}$ and $\left\{\varepsilon_{l}\right\}$ are eigenfunctions and eigenvalues of the electronic part of the Hamiltonian $H(t)$ at a given time $t_{j}$.

The lattice equations are written as

$$
\begin{aligned}
u_{i}\left(t_{j+1}\right) & =u_{i}\left(t_{j}\right)+\dot{u}_{i}\left(t_{j}\right) \Delta t \\
\dot{u}_{i}\left(t_{j}+1\right) & =\dot{u}_{i}\left(t_{j}\right)+\frac{F_{i}\left(t_{j}\right)}{M} \Delta t .
\end{aligned}
$$

Hence, the electronic wave functions and the displacement coordinates at $j+1$-th time step are obtained from the $t$-th time-step. Periodic boundary conditions are assumed for the electronic wave functions, $\psi_{k, s}$, and the lattice displacement, $u_{i}$.

The position, energy and charge are written at every 1000 time steps. A time step of $\Delta t=0.008$ femtosecond is used. We follow the dynamics of systems up to 400 femtoseconds.

\section{RESULTS OF SIMULATIONS}

We have considered polyacetylene chains with $N=104 \mathrm{CH}$ groups in all simulations. One single positive charged polaron 

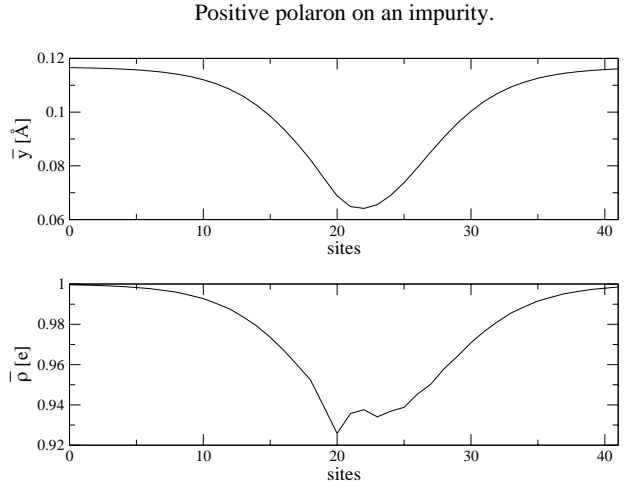

FIG. 2: Static fully self consistent solution of a polaron trapped in an impurity. Can be observed that there is a displacement between the polaron center (22-th site) and the impurity (20-th).

is initially located around the 22-th site. The electric field is turned on in the beginning of the simulation and after 60 femtoseconds it is switched off. An adiabatic switching is used to turn on and off the electric field. The electronic field values range from $|E|=0.005 E_{0}$ to $|E|=0.03 E_{0}$, where $E_{0}=$ $\frac{\hbar \omega_{Q}}{e a}=1.3 \times 10^{7} \mathrm{~V} / \mathrm{m}$.

The calculated energies in each simulation are the electronic energy, the lattice potential energy, and the lattice kinetic energy. The lattice total energy is the sum of these energies. We calculate the effective potential from the difference between the translational kinetic energy of the free polaron case and the case with bonding radicals [12]. This assumption is found to be a good approximation, despite some kinetic energy goes to lattice oscillations when the polaron accelerates.

To analyze the polaron graphs we have used an order parameter $\bar{y}_{i}$ to describe the sites displacement and a mean value to the charge density $\bar{\rho}_{i}$.

$$
\begin{array}{r}
\bar{y}_{i}=(-1)^{i} \frac{-y_{i-1}+2 y_{i}-y_{i+1}}{4} \\
\bar{\rho}_{i}=\frac{\rho_{i-1}+2 \rho_{i}+\rho_{i+1}}{4}
\end{array}
$$

The polaron position is calculated from a cyclic mean value of the order parameter.

$$
\langle p\rangle=\frac{N}{2 \pi}\left[\sum_{n=1}^{N} p_{n} \exp \left(\frac{i 2 \pi}{n}\right)\right] .
$$

The deviation of the polaron center from the impurity can be observed in Fig. 2. This deviation can be explained by the fact that charge concentrates on even sites in the left side of the polaron and on odd sites in the right side of the polaron.

The potential that holds the polaron is asymmetric with respect to the impurity site. In the case which the polaron is initially trapped in an impurity, when an electric field is applied, there is a polaron charge oscillation around the impurity site. As the impurity is located in the left side, the energy that the

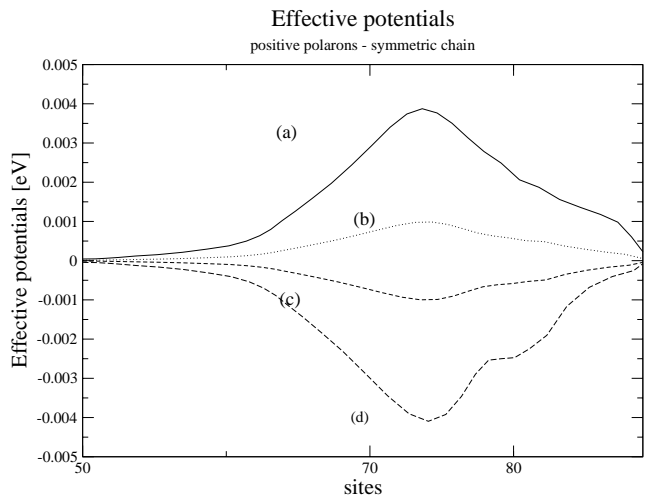

FIG. 3: Effective potentials of a positive polaron due to impurities in a symmetric chain. (a) $V_{p}=-0.03$, (b) $V_{p}=-0.01$, (c) $V_{p}=+0.01$, (d) $+V_{p}=0.03$.

polaron have to receive from the electric field to untrap itself and run to left side (positive electric field) is greater than the energy needed to untrap and run to the right side (negative electric field). In polaron dynamics, there is dissipation of polaron energy, caused by the electron phonon interaction. There are two kinds of potentials generated by impurities: considering positive polarons, a donor impurity $\left(V_{p}>0\right)$ generates an attractive potential well and an acceptor impurity $\left(V_{p}<0\right)$ generates a repulsive potential barrier. For negative polarons the converse is observed.

Considering the chains with no symmetry breaking interaction $\left(\delta_{0}=0\right)$ we calculate effective potentials for positively charged polarons due to impurities. The potentials were calculated to $V_{p}=-0.03 t_{0},-0.01 t_{0},+0.01 t_{0}$ and $+0.03 t_{0}$. They are shown in Fig. 3. All impurities are located on site 81. Fig. 3 shows that the strongest interactions with impurities occur close to site 74 . The maximum and the minimum values of the effective potentials are located at this site, showing that the potential felt by a polaron due to impurities is displaced from the impurity position. The effective potentials also allow to conclude that the interaction between polarons and impurities ranges about 32 sites.

To asymmetric chains $\left(\delta_{0} \neq 0\right)$ the effective potentials were also calculated and are showed in Fig. 4. The same impurities parameters were used to calculate these potentials and impurities also were located on site 81 . In figure 4 it can also be observed the asymmetry of effective potentials. The strongest interaction occurs close to site 77 . The interaction range is about 28 sites.

Comparing the impurity action on polarons in symmetric and asymmetric chains we have found that in the asymmetric chains the impurity action is very strong, because the maximum and the minimum values of the effective potentials are more accentuated. Nevertheless, in asymmetric chains the interaction range between polaron and impurities is very short as compared to symmetric chains. However, the main features of the impurity-polaron interactions are the same in both cases. 


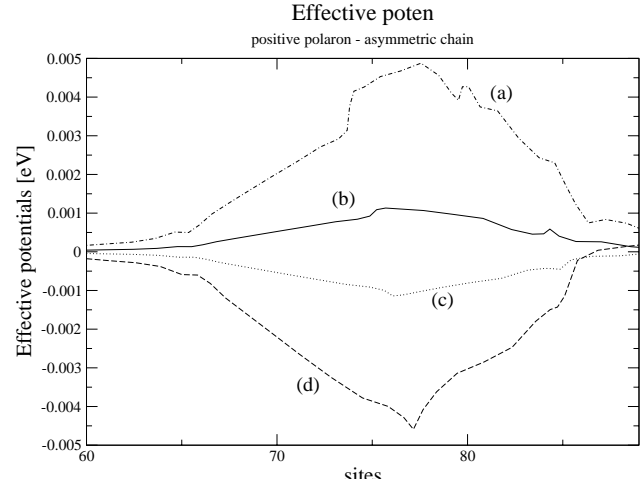

FIG. 4: Effective potentials of a positive polaron due to impurities in a asymmetric chain. (a) $V_{p}=-0.03$, (b) $V_{p}=-0.01$, (c) $V_{p}=$ $+0.01,(\mathrm{~d})+V_{p}=0.03$.

\section{CONCLUSION}

We have investigated the propagation of polarons on a single conjugated polymeric chain in the presence of impurities. This study was carried out through numerical calculations. A modified SSH type Hamiltonian considering an external electric field, Coulomb interactions and impurities has been used. The initial configuration is fully self consistent and the time evolution of the system is carried out using the Schrödinger and the Euler-Lagrange equations.

The polaron that was initially trapped on an impurity feels an effective potential which is asymmetric with reference to impurity site position. The energy received by the polaron from the electric field determines as the polaron passes or remains trapped.

For positive charged polarons, a negative impurity parameter $V_{p}$ generates a repulsive potential and a positive impurity parameter generates an attractive potential. For negative polarons the converse is observed.

To the same impurity, the potential felt by the polaron is very high in the asymmetric case. Therefore, in the symmetric one, the polaron scattering is less intense. As a result, the mobility of the charge, and hence its transportation, is more favorable in symmetric chains.

\section{ACKNOWLEDGMENTS}

We thank CAPES and CNPQ for financial support.
[1] G. M. e Silva and P.H. Acioli, Synth. Met. 87, 249 (1997).

[2] G. M. e Silva and P.H. Acioli, J. Mol. Struct. 464, 67 (1999).

[3] C. da Silva Pinheiro, P. H. Acioli, and G. M. e Silva, J. Mol. Struct. 539, 55 (2001).

[4] R. A. English, Z. L. Miskovic, S. G. Davison, and F. O. Goodman, Phys. Rev. B 54, 10 (1996), 54, 254 (1996).

[5] W. Hu, H. Nakashima, K. Furukawa, Y. Kashimura, K. Ajito, and K. Torimitsu, Appl. Phys. Lett. 85, 115 (2004).

[6] W. P. Su, J. R. Schrieffer, and A. J. Heeger, Phys. Rev. B 22 , 2099 (1980), 28, 1138 (1983).
[7] S. A. Brazovskii, N. N. Kirova, Pis'ma Zh. Eksp. Teor. Fiz. 33, 6 (1981) (JETP lett. 33, 4 (1981)).

[8] A. Terai and Y. Ono, J. Phys. Soc. Jpn 55, 213 (1986).

[9] Z. Na, C. Q. Wu, and X.Sun, Phys. Rev. Lett. 93 (21), 216407 (2004).

[10] A. A. Johansson and S. Stafstrom, Phys. Rev. B 69 (23), 235205 (2004).

[11] Y. H. Yan, Z. An, and C. Q. Wu, Eur. Phys. J. B 42, 157 (2004)

[12] G. M. e Silva, Phys. Rev. B 6110777 (2000). 